\title{
Insights into the immuno-pathogenesis of acute respiratory distress syndrome
}

\author{
Judith Ju Ming Wong ${ }^{1,2}$, Jing Yao Leong ${ }^{2}$, Jan Hau Lee $^{1}$, Salvatore Albani ${ }^{2,3}$, Joo Guan Yeo ${ }^{2,3}$ \\ ${ }^{1}$ Children's Intensive Care Unit, Department of Pediatric Subspecialty, KK Women's and Children's Hospital, Singapore; ${ }^{2}$ Translational Immunology \\ Institute, Singhealth/Duke-NUS Academic Medical Centre, Singapore; ${ }^{3}$ Division of Medicine, KK Women's and Children's Hospital, Singapore \\ Contributions: (I) Conception and design: JJM Wong, JG Yeo; (II) Administrative support: None; (III) Provision of study materials or patients: None; \\ (IV) Collection and assembly of data: JJM Wong, JY Leong; (V) Data analysis and interpretation: All authors; (VI) Manuscript writing: All authors; \\ (VII) Final approval of manuscript: All authors. \\ Correspondence to: Judith Ju Ming Wong. Children's Intensive Care Unit, Department of Pediatric Subspecialty, KK Women's and Children's Hospital, \\ 100 Bukit Timah Road, Singapore. Email: judith.wong.jm@singhealth.com.sg.
}

\begin{abstract}
Acute respiratory distress syndrome (ARDS) is a clinical syndrome associated with oxygenation failure resulting from a direct pulmonary or indirect systemic insult. It is a complex etiological phenomenon involving an array of immune cells acting in a delicate balance between pathogen clearance and immunopathology. There is emerging evidence of the involvement of different immune cell types in ARDS pathogenesis. This includes polarization of alveolar macrophages (AMs), neutrophil netosis, the pro-inflammatory response of $\mathrm{T}$ helper 17 subsets, and the anti-inflammatory and regenerative role of $\mathrm{T}$ regulatory cell subsets. Knowledge of these pathogenic mechanisms has led to translational opportunities, for example, research in the use of methylprednisolone, DNAse, aspirin, keratinocyte growth factor and in the development of stem cell therapy for ARDS. Discovering subgroups of patients with ARDS afflicted with homogenous pathologic mechanisms can provide prognostic and/or predictive insight that will enable precision medicine. Lastly, new high dimensional immunomic technologies are promising tools in evaluating the host immune response in ARDS and will be discussed in this review.
\end{abstract}

Keywords: Respiratory; lung; acute lung injury; immunology; immunity; immune cells

Submitted Mar 12, 2019. Accepted for publication Aug 26, 2019.

doi: $10.21037 /$ atm.2019.09.28

View this article at: http://dx.doi.org/10.21037/atm.2019.09.28

\section{Introduction}

Acute respiratory distress syndrome (ARDS) is a clinical syndrome characterized by oxygenation failure with significant morbidity and mortality due to inflammation and accumulation of a proteinaceous exudate within the pulmonary air spaces $(1,2)$. Its most common etiology is respiratory infection due to viruses and bacteria with the occasional fungal pathogens but it can also be the result of an indirect sepsis or sterile systemic inflammation due to burns, polytrauma and pancreatitis (2). The pathogenesis of ARDS is multi-factorial and involves diverse immune cells of both the innate and adaptive immune system that mediate the propagation of lung injury triggered by these insults (3). In this review, we will critically appraise the roles of the different immune cell types in ARDS pathogenesis with a focus on recent studies which utilize high dimensional immunomics technologies. This multiparametric approach is well-suited to address the complexity of ARDS immunopathogenesis in a comprehensive and holistic manner.

\section{Innate immune system}

Alveolar epithelial cells (AECs) which form the pulmonary epithelium act as a physical barrier to the external environment (4). Alveolar macrophages (AMs) residing within the air spaces and interstitium are the earliest immune 
cells to detect the presence of pathogens or injury (4). In contrast, dendritic cells (DCs) act as an interface between the innate and adaptive immune system and resides deep to the airway epithelial layer, within alveolar septae and pulmonary interstitium but has extensive dendrites that are apt in sensing its environment for inhaled antigens (4). DCs are professional antigen presenting cells that will mature and migrate to the lymph nodes to prime the adaptive responses when exposed to a presentable antigen (5). Collectively, AECs, AMs and DCs function as sensor cells which detect danger signals through the pattern recognition receptors (PRR) and initiate innate responses. These danger signals include those that are (I) infective in origin; pathogen associated molecular patterns (PAMPs), and (II) non-infective from cellular damage; danger associated molecular patterns (DAMPs) or alarmins (6). Their ability to sense these signals are attributed to several receptor pathways including Toll-like receptors, Retinoic acid inducible gene 1-like receptors, cytosolic DNA sensors and nucleotide oligomerization domain-like receptors $(4,6)$.

Activated sensor cells directly recruit effector cells for pathogen clearance and secrete a first wave of cytokines (AECs secrete IFN $\lambda$, CCL2, AMs secrete IFN $\alpha$, IFN $\beta$, IL-6, TNF, IL-12 and DCs secrete IL-12, IL-23, IL1 $\beta$ ) to alert resident lymphocytes (4). These tissue resident lymphoid cells consisting of cytotoxic T cells, TH1 cells, TH17 cells, NK cells, NKT cells, $\gamma \delta \mathrm{T}$ cells and innate lymphoid cells (ILCs) (type 1,2 and 3) respond to the first wave of cytokines by secreting a second wave (7). Group 1 ILCs secrete TH1 cytokines (IFN $\gamma$ and TNF), group 2 ILCs secrete TH2 cytokines (IL-5, IL-13, IL-33) whereas group 3 ILCs secrete TH17 cytokines (IL-17A and IL-22) $(7,8)$. Cytotoxic T cells become primed and increases its cytotoxic activity. NK cells produce IFN $\gamma$ and IL-22 exerting cytotoxic effects on viral and intracellular bacteria infected cells and promote DC maturation. $\gamma \delta \mathrm{T}$ cells are a major source of IFN $\gamma, \mathrm{TNF} \alpha$ and IL-17. These second wave of cytokines, are chemoattractant towards circulating neutrophils and monocytes, stimulate AECs to increase production of antimicrobial peptides and chemokines, and $\mathrm{AMs}$ to enhance phagocytosis. In summary, a constellation of immune cells acts in concert through cyclical waves of cytokines within the lung interphase.

\section{Diverging roles of AMs in ARDS}

In health, AMs performs its primary role in tissue homeostasis through the scavenging and clearance of cellular debris and apoptotic cells without inciting an inflammatory response (9). This includes secretion of GM-CSF for surfactant catabolism, without which surfactant will accumulate in the alveoli causing impaired gas exchange and pulmonary alveolar proteinosis (10). In this homeostatic state, AMs receive constant negative regulatory signals including CD200, TGF $\beta$ and IL-10 from the surrounding AECs, which prevents the AMs from being activated (11,12). A high basal CD 200 receptor expression by myeloid cells in contact with the airway mucosa allows it to deal with the amount of pathogen exposure (11). CD 200 ligand binding results in an inhibitory signal to the CD 200 receptor cells, therefore maintaining myeloid restraint (11). TGF $\beta$ and IL-10, both enhances CD 200 receptor expression, thus increasing the sensitivity of myeloid cells towards CD200 mediated inhibition. Separately, TGF $\beta$ acts in concert with epithelial $\alpha_{v} \beta_{6}$ integrin to suppress AM activation and type 1 IFN signaling in an influenza acute lung injury (ALI) model (13).

During the disease course of ARDS, AMs exist in a dynamic balance between pro-inflammatory M1 and antiinflammatory M2 polarized states. Circulating monocytes are proinflammatory but AMs were shown to be antiinflammatory in a lipopolysaccharide (LPS) induced ALI mouse model (14). In this study, depletion of circulating monocytes reduced neutrophilic infiltration and inflammatory mediator expression e.g., IL-17, MCP-1, HMGB-1 (14). In contrast, depletion of AMs upregulated these mediators, suggesting its protective role (14). Clinical studies of patients with direct and indirect ARDS reaffirmed that a state of sustained monocyte influx positively correlates with severity of respiratory failure as indicated by the higher oxygenation index (15). Further studies describe M1 and M2 macrophage subtypes with distinct surface markers (M1 associated with ICAM-1, CD 40, and M2 with transferrin receptor, mannose receptor), gene expression and effector function (16). The M1 subtype is induced by proinflammatory TH1 cytokines, such as IFN- $\gamma$ as well as LPS (16). It then responds with the secretion of IL-1 $\beta$, IL-12, TNF $\alpha$ and inducible nitric oxide synthase (16). In contrast, the M2 subtype is induced by TH 2 cytokines IL- 4 and IL-13 and secretes anti-inflammatory IL-10 (16). These polarized states occur in flux during the course of ALI (16). Intratracheally administered LPS in conjunction with M2 AMs resulted in reduced neutrophilic infiltration, reduced oxidative stress and TNF $\alpha$, IL-1 $\beta$, IL-6, and increased T regulatory (Treg) cells with IL-10 compared to LPS alone (17). Blockade of programmed death ligand 1/programmed death 
1 (PD-L1/PD-1) pathway reversed cytokine production of M2 AM in bronchoalveolar lavage (17).

Treatment with methylprednisolone promoted M2 rather than $M 1$ polarization which aided in the preservation of lung mechanics (18). This was demonstrated in a mouse model of sepsis and ARDS where methylprednisolone administered intravenously to mice together with LPS was associated with induction of M2 phenotype and cytokine profile in a dose dependent manner (18). Induced M2 cells co-cultured with $\mathrm{CD}^{+}$naïve $\mathrm{T}$ cells, in turn, induce differentiation of Treg lymphocytes demonstrating for the first time, macrophage-Treg interactions (18).

\section{Implications of netosis/neutrophils in ARDS}

Neutrophils patrolling in the blood stream and marginated in the pulmonary vasculature constitute a ready pool of effector cells that migrate towards the presence of chemoattractants like CXCL8 secreted by sensor cells (19). To reach the pathogenic site within pulmonary tissue, neutrophils secrete MMP9 which breaks down the collagen within the extra cellular matrix (ECM) (20). The presence of TNF in the surrounding tissue enhances MMP9 secretion by neutrophils thereby directing them towards the focus of inflammation/infection (21). Neutrophils subsequently begin phagocytosis and degranulation of neutrophil elastase, myeloperoxidase, reactive oxygen species (ROS) and neutrophil extracellular traps (NETs) which assist in clearance of viral, bacterial or fungal pathogens. Netosis refers to a neutrophil specific cell death process whereby NETS are deployed to immobilize and kill pathogens while precipitating its own death (22). NETs consist of histones, decondensed chromatin fibres and granule components. While its main purpose is to limit the pathogenic burden, some bacteria and viruses cause excessive NETs formation which leads to further lung damage and respiratory failure $(23,24)$.

Lethal doses of Influenza A used in mice incites extensive NETs formation within the alveoli resulting in airway obstruction within bronchioles, extending to endothelial damage thus impairing gas exchange across blood vessels (25). Respiratory syncytial virus (RSV) invoked NETs formation that normally affords for successful capture of RSV particles in vitro, thereby preventing infection of target epithelial cells (24). However, in vivo, marked formation of NETs observed during RSV lower respiratory tract infection resulted in accumulation of dense
NETs plugs which obstructed the airways without capturing of viral antigen, suggesting an exaggerated adverse and ineffective response (24).

Certain bacteria like staphylococcus aureus, streptococcus pneumoniae and hemophilus influenzae have been described to evade the bactericidal activity within NETs $(23,26)$. Partially degraded NETs were observed when incubated with streptococcus pneumoniae, suggesting degradation of DNA in NETs by endonucleases produced by streptococcus pneumoniae (23). Moreover, co-infections induced larger yet ineffective clusters of NETs compared to bacteria or Influenza alone, and was associated with increased respiratory failure, inflammation, and bacteremia (23). These NETs formation entangled within alveoli in areas of histological tissue injury and bronchoalveolar fluid provides evidential link between netosis and ARDS $(25,27)$.

In transfusion-related acute lung injury (TRALI), activated platelets induce NETs formation promoting coagulation and thrombi formation in the lungs $(28,29)$. In a patient with TRALI, NETs were detected within the lung microvasculature and traces of the NETs' components were detected in circulation (28). This was in contrast with a patient who developed transfusion associated circulatory overload (TACO), in which neutrophils were found in the alveoli without accompanying NETs formation (28), providing evidence on the immunological differences between TRALI and TACO. The same study noted higher amounts of plasma NETs components in TRALI patients as opposed to other causes of lung injury (28). Distinguishing TRALI and TACO through better markers will facilitate the differential treatment required.

As human neutrophils generate NETs in the presence of platelets activated by thrombin receptor-activating peptide (TRAP), investigators attempted to inhibit platelet activation with tirofiban (glycoprotein IIb/IIIa inhibitor) (28). In this mouse model of TRALI, tirofiban decreased to NETs formation, extravascular lung water, lung vascular permeability and platelet sequestration (28). In a clinical trial with healthy human volunteers randomized to low dose aspirin, high dose aspirin or placebo prior to inhalation of LPS (30). Aspirin (regardless of dose) reduced neutrophil counts and neutrophil proteases (MMP8, MMP9) in the bronchoalveolar lavage fluid (30). However in patients admitted to the emergency department who were at risk of developing ARDS, aspirin was not observed to prevent the development of ARDS in a clinical phase $2 \mathrm{~b}$ randomized controlled trial (31). 


\section{Adaptive immune system}

Adaptive responses are triggered shortly after activation of the innate system. In response to the first wave of cytokines, pulmonary DCs migrate to regional lymph nodes and prompts activation, proliferation and differentiation of naïve $\mathrm{CD}^{+} \mathrm{T}$ cells to a variety of effector $\mathrm{CD}^{+}$cells. The CD4 adaptive response is polarized based on whether TH1 or TH2 responses are generated. TH1 differentiation occurs under the influence of T-Bet, STAT4 (transcription factors), and IL-12, and is characterized by IFN $\gamma$ induced cell death in infected cells. TH2 differentiation requires GATA3, STAT6 (transcription factors), IL-4 and is characterized by stimulation of $\mathrm{B}$ cell differentiation and antibody production.

In response to $\mathrm{DC}$ antigen presentation, $\mathrm{CD}^{+} \mathrm{T}$ cells also differentiate into cytotoxic or memory $\mathrm{CD}^{+} \mathrm{T}$ cells. LPS induction of ALI mice resulted in $\mathrm{CD}^{+} \mathrm{T}$ cell lung infiltration by the 12 hours window (32). Patients with viral lower respiratory tract infections have higher CD8: CD4 cell ratios in airway aspirates compared to patients with no infection; a phenomenon particularly pronounced in patients with infective ALI (33). $\mathrm{CD}^{+} \mathrm{T}$ cells recognize antigen bound MHC complexes via $\mathrm{T}$ cell receptors effecting (I) perforin/granzyme mediated cytolysis, (II) FasL/Fas signaling and (III) TRAIL/TRAIL-DR signaling. Perforin first form pores and then granzyme is released into the target cell inducing apoptosis. FasL binding to Fas receptor and TRAIL ligand binding to TRAIL-DR receptor on infected cells triggers apoptosis. Additionally, cytotoxic T cells secrete IFN $\gamma$, TNF $\alpha$ and IL-2 (induces proliferation and survival of cytotoxic $\mathrm{T}$ cells). In combination, these cytokines have extremely potent cytotoxicity. Along with Treg cells, cytotoxic $\mathrm{T}$ cells also produce IL-10, without which, its own inflammatory actions may be overexuberant. Memory T cells are also capable of IL-22, IL-17 and IFN $\gamma$ secretion independent of $\mathrm{T}$ cell receptor stimulation which contribute to ALI (34).

\section{Inflammatory $T h-17$ response in selected ARDS patients}

TH17 cells are the prominent producers of IL-17A, IL17F, IL-2 1 and IL-22. The hallmark cytokine IL-17 triggers downstream production of chemokines CXCL9 and CXCL10 to recruit neutrophils and increases bacterialinduced neutrophilic response in the bone marrow. Indeed, the absence of IL-17A in LPS induced ALI resulted in a 100-fold reduction in neutrophil infiltration (35).

LPS induced ARDS in mice led to clonal expansion of
TH 17 cells in the lungs which was associated with increased alveolar epithelial permeability (36). Deficient TH17 cells, loss of IL-17 receptor C or anti-IL-17A antibody decreased alveolar epithelial permeability (36). Direct addition of recombinant IL-17A increased permeability in human alveolar epithelial monolayers (36). Another study showed that inhibition of IL-17 and IL-22 resulted in decreased recruitment of neutrophils and macrophages, and increasing numbers of Treg cells (37). This was also corroborated in human clinical studies, where bronchoalveolar lavage (BAL) from adult ARDS patients showed increased IL-17A concentrations (36). BAL from children with sepsis induced ALI $(n=50)$ had increased TH17 cells in patients with greater severity of illness and in non-survivors (38). Patients with pulmonary complications of trauma had increased TH17 cell cytokines including IL-17A, IL-17F, IL-21 and IL-6 (39). However, analysis of these lung trauma patients indicated no difference in patients with/without ARDS, except that plasma and bronchoalveolar lavage IL-6 was markedly greater in patients who developed ARDS (39). Though evidence indicate that TH17 responses in the lung are inflammatory, the presence of IL-22 can contribute towards lung epithelial proliferation and repair (40).

\section{Suppressive and regenerative role of $T$ regulatory cells in ARDS patients}

Treg cells as defined by the expression of CD25 and the hallmark transcription factor FOXP3, serve to restrain excessive inflammation and restore homeostasis by secreting IL-10 and TGF $\beta$. An LPS induced ALI mouse model demonstrated that depletion of Treg cells by intraperitoneal injection of either anti-CD25 or TGF $\beta$ neutralization resulted in prolonged inflammation dominated by neutrophils, macrophages and TH17 cells (37). Treg cells and IL-10 was also found to be protective in a TRALI mice model (41).

Clinical studies comparing patients with ARDS, pneumonia and healthy controls showed increased systemic and pulmonary IL-10 producing $\mathrm{CD}^{+} \mathrm{T}$ cells in the ARDS group (42). ARDS survivors also had higher IL-10 producing $\mathrm{CD}^{+} \mathrm{T}$ cells compared to non-survivors (42). The antiinflammatory effects of Tregs are ascribed to elimination of myeloid antigen presenting cells (monocytes and DCs) by granzyme $\mathrm{B}$ production and suppression of $\mathrm{CD} 8^{+} \mathrm{T}$ cell IFN $\gamma$ production (42). Th-17/T reg ratio has also been studied as a biomarker for mortality prediction (43). The ratio increased from mild to severe ARDS within 
24 hours of ARDS, positively correlated with Acute physiology, Age, Chronic Health Evaluation (APACHE) II score, Sequential Organ Failure Assessment (SOFA) score and Lung Injury Score, and negatively correlated with partial pressure of arterial oxygen: fraction of inspired oxygen (PF) ratio (43). It predicted mortality in a cohort containing patients with ARDS and healthy control with an area under the receiver operating curve of 0.79 (43). In healthy volunteers exposed to LPS instillation $(\mathrm{n}=15)$, BAL taken across a $24 \mathrm{hr}$ window revealed $\mathrm{CD}^{+}$and $\mathrm{CD} 8^{+} \mathrm{T}$ cells had increased activation markers (HLA-DR ${ }^{+}$DR $38^{+}$), decreased naïve markers $\left(\mathrm{CD} 45 \mathrm{RA}^{+} \mathrm{CD} 27^{+} \mathrm{CCR} 7^{+}\right)$, increased effector memory markers (CD45 $\mathrm{RA}^{-} \mathrm{CD} 27^{+}$ $\mathrm{CCR}^{-}$) and importantly increased Treg cells (44).

Interestingly, Treg cells also aid in regeneration. LPS exposure elicits alveolar epithelial damage which then undergoes proliferation and repair. In mouse models, this coincides with increased Treg cells. Treg cell depletion decreases alveolar epithelial proliferation and delays recovery (45). Adoptive transfer of Treg cells back into lymphocyte deficient $\mathrm{Rag}^{-/-}$mice corrects not only elevated alveolar proinflammatory cytokines and increased neutrophil apoptosis, but also normalized resolution of lung injury $(46,47)$. Proliferation of alveolar epithelium is enhanced by keratinocyte growth factor (KGF) expressed by Treg during the recovery phase of ALI (48). When Tregs were isolated specifically from the pulmonary environment and co-cultured with AECs, both growth factors, KGF and amphiregulin were produced (48).

In light of the beneficial effects mediated by Treg cells, several studies to boost their frequency or function are underway in animal models. DNA methyltransferase (DNMT) inhibitor augments Treg cell number and function by enhancing FOXP3 expression (49). In an LPS induced ALI mouse model, DMNT inhibitor increases lung Treg cells and accelerates repair (49). The macrolide antibiotic, erythromycin, when given to rats exposed to lung inflammation induced by cigarette smoke, was shown to increase the number of Treg cells and inhibit lung damage through yet an unknown mechanism (50). Acting on the cAMP signaling pathway and possibly via modulation of transcription factors (FOXP3, ROR $\gamma \mathrm{t}$, STAT3), phosphodiesterase inhibitors increase the number of Treg cells, restoring the Treg/Th17 ratio (51). This was associated with attenuation of lung injury and reduced mortality in mice with ARDS induced through cecal ligature and puncture (51).
Age-related differences exist in Treg cells and in particular, their response to ALI during early postnatal development was shown in mice models (52). 72 hours after LPS exposure, neonatal lungs were slower to resolve inflammation and expressed lower mRNA levels of CCL2, CCL5, CXCL10, and IL-10 as compared with juvenile mice (52). Neonatal mice also were not able to significantly increase airway Treg cells as opposed to juvenile mice (52). When adult Treg cells were adoptively transferred into neonatal mice, they had reduced lung inflammation and improved weight gain (52).

PD1 is an immune checkpoint protein involved in the suppression of chronic $\mathrm{T}$ response and can directly modulate the ability of Treg cells to suppress ARDS. PD1 deficient mice seemed to lose the beneficial effects of Treg cells on lung inflammation, however, when exogenous $\mathrm{T}$ reg cells were adoptively transferred, they were able to home in to the injured lung tissue to decrease neutrophil influx, decrease lung cell apoptosis and increase regional IL-10 levels (53). The PD-L1, rather than its receptor PD1 was later shown to be crucial in influencing response of Treg cells (54). Cytotoxic T cell associated antigen 4 (CTLA4), another immune checkpoint protein, commonly expressed on Tregs, that acts as a negative $\mathrm{T}$ cell proliferation regulator during the early phase of immune response was investigated in patients with ALI (55). CTLA4 on $\mathrm{CD}^{+}$and $\mathrm{CD} 8^{+} \mathrm{T}$ cells were elevated in patients with ALI compared to controls and there was positive correlation with the APCAHE II score (55). At the time of death, expression CTLA4 was higher than at the time of recruitment (55).

Lastly, the immunomodulatory effects of Treg cells and M2 polarized AMs, is asserted as one of the main mechanisms for the beneficial effects of cell-based therapy on ARDS, specifically mesenchymal stem cell (MSC) therapy (56). It is shown to enhance resolution of inflammation $(57,58)$, restore alveolar-capillary barrier and lung function $(57,59)$ and improve pathogen clearance $(59,60)$. On the back of convincing preclinical studies, a phase 1 trial studying the effects of a single dose of intravenous MSCs in patients with moderate-to-severe ARDS determined this therapy to be safe and potentially beneficial in reducing the lung injury score and sequential organ failure assessment score in a dose dependent manner (61). Following that, the recent Stem cells for ARDS Treatment (START) trial, a randomized phase 2a trial $(\mathrm{n}=60)$, reaffirmed safety in the usage of MSC therapy, but was underpowered to detect any clinical improvement (62). 


\section{Mass cytometry reveals Involvement of $\gamma \delta T$ cells in bacterial lung infection}

$\gamma \delta \mathrm{T}$ cells are resident tissue lymphocytes involved in early primary effector response activating neutrophils, macrophages and cytotoxic T cells. Cytometry by timeof-flight (CyTOF) mass cytometry was used to interrogate BAL, spleen and lymph nodes from streptococcus pneumoniae infected/uninfected mice to identify $\gamma \delta \mathrm{T}$ cell responses (63). Active infection induces an increase in lung $\gamma \delta \mathrm{T}$ cells and the local production of IFN $\gamma$ and IL-17 (63). Novel Gr1 ${ }^{+} \mathrm{CD} 14^{+}$ markers identified were able to differentiate IL-17 from IFN $\gamma$ producing $\gamma \delta$ T cell (63). Phosphorylation of ZAP70 and $\mathrm{p} 38$ in $\mathrm{Gr}^{+} \gamma \delta \mathrm{T}$ cells suggest that both innate and $\mathrm{T}$ cell receptor signaling pathways are involved in $\gamma \delta \mathrm{T}$ cell activation, whereas, phosphorylation of ZAP70 alone in Gr1$\gamma \delta \mathrm{T}$ cells suggest activation is $\mathrm{T}$ cell receptor dependent (63).

\section{Exploiting technological opportunities in ARDS}

One of the main limitations as reflected by the current literature on ARDS is the paucity of basic and translational studies on healthy or diseased human subjects. As described above, majority of studies were performed on well-defined animal models of ALI. Another limitation is the low dimensionality of techniques deployed to phenotype cells, activation states and cytokines profiles.

One recent technological development in the field of immunology is the emergence of CyToF (64). Cellular subsets are stained with rare heavy metal isotopes conjugated to antibodies targeting cellular markers of interest. These stained cells are channeled towards a neubuliser and vaporized into its constituent ions and detected for the presence of those rare heavy metals in a mass spectrometry configuration. As CyTOF allows for the staining of approximately 40 cell markers without the need for spectral compensation, it has the potential to identify new cell populations which would not otherwise be uncovered by conventional flow cytometry (64). Recently, CyTOF mass cytometry investigation of AMs identified 3 distinct novel subtypes (65). $\mathrm{CD} 33^{+} \mathrm{CD} 71^{+} \mathrm{CD} 163^{+} \mathrm{AMs}$ from bronchoalveolar lavage from healthy, non-ARDS and ARDS subjects were interrogated and CD169 and PD-L1 surface expression produced the best discrimination between these subtypes $\left(\mathrm{CD} 169^{\text {hi }} \mathrm{PD}-\mathrm{L} 1^{\text {hi }} \mathrm{AM}-1, \mathrm{CD} 169^{\text {hi }} \mathrm{PD}-\mathrm{L} 1^{\text {lo }}\right.$ AM-2 and CD169 ${ }^{\text {lo }}$ AM-3) (65). In view of this finding, PDL1 gene expression was evaluated and subjects with high ventilator-free days were found to express higher PD-L1 in two independent cohorts (65).

Given the heterogenous etiology of ALI/ARDS and the complex host immune response, CyTOF is a wellsuited tool capable of interrogating a variety of cell types simultaneously. Nevertheless, the uptake rate of CyTOF for cell characterization in patients with ALI/ARDS is still limited due to the prohibitive cost of acquiring the machine and complexity of high dimensional analysis.

Another promising strategy is the next generation sequencing technique, RNA-Seq, which quantifies the transcriptome of a cell or tissue thus capturing a snapshot of gene expression of that cell in the current disease state (66). In contrast to analyzing cells or tissue in bulk, single cell approaches now allow for the interrogation of the transcriptome at single cell resolution (67). Isolation of single cells and amplification of the genome is necessary prior to analysis (67). Single-cell RNA-seq methods allows measurement of expressed transcripts in rare or poorly defined cell populations hence providing the opportunity to evaluate gene expression in potential individual cell populations driving ARDS pathogenesis. This technique allows comparison between cells or tissues in disease states to identify disease-relevant genes and pathways.

\section{Conclusions}

ARDS is a complex etiological phenomenon involving a complex array of immune players acting in concert. Emerging evidence reveal the importance of AMs, neutrophils, Tregs and Th17 subsets as potential key players (Table 1). At this moment, it is hard to decipher the relative importance of the key players involved in the pathology of ARDS. Yet with the development of new single cell technologies, we may be better positioned to dissect through this heterogeneous cloud of immune network, and unveil distinct subsets that may allow for new diagnostic and therapeutic opportunities. 
Table 1 Summary of the main immune cell types involved in acute respiratory distress syndrome and their immunopathogenesis

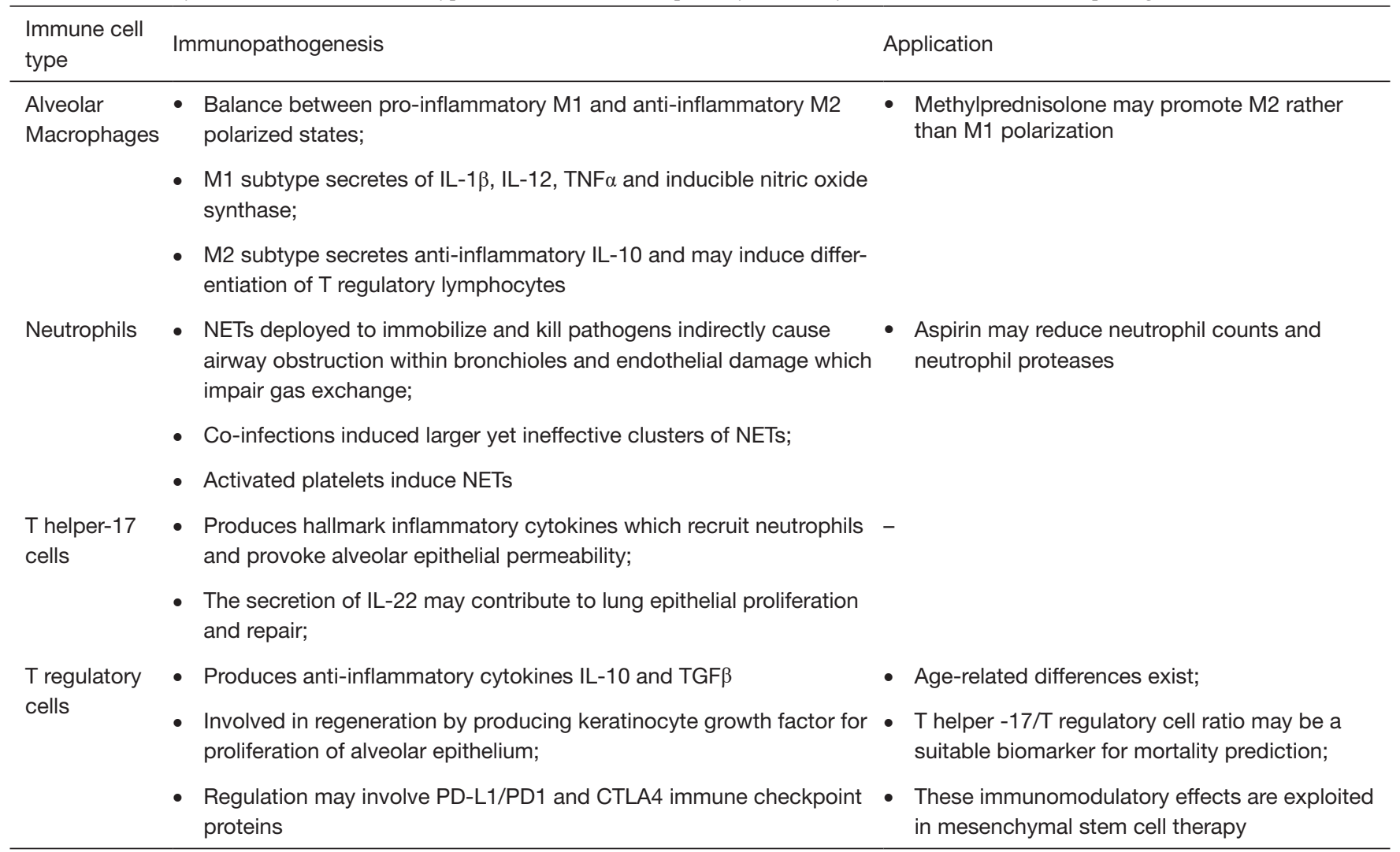

NETs, neutrophil extracellular traps; PD, programmed death; PD-L1, programmed death ligand 1; CTLA4, cytotoxic T cell associated antigen 4 .

\section{Acknowledgments}

None.

\section{Footnote}

Conflicts of Interest: The authors have no conflicts of interest to declare.

Ethical Statement: The authors are accountable for all aspects of the work in ensuring that questions related to the accuracy or integrity of any part of the work are appropriately investigated and resolved.

\section{References}

1. Ashbaugh DG, Bigelow DB, Petty TL, et al. Acute respiratory distress in adults. Lancet 1967;2:319-23.

2. Bernard GR, Artigas A, Brigham KL, et al. The
American-European Consensus Conference on ARDS. Definitions, mechanisms, relevant outcomes, and clinical trial coordination. Am J Respir Crit Care Med 1994;149:818-24.

3. Yang CY, Chen CS, Yiang GT, et al. New Insights into the Immune Molecular Regulation of the Pathogenesis of Acute Respiratory Distress Syndrome. Int J Mol Sci 2018. doi: 10.3390/ijms19020588.

4. Iwasaki A, Foxman EF, Molony RD. Early local immune defences in the respiratory tract. Nature reviews Immunology 2017;17:7-20.

5. Worbs T, Hammerschmidt SI, Forster R. Dendritic cell migration in health and disease. Nat Rev Immunol 2017;17:30-48.

6. Newton K, Dixit VM. Signaling in innate immunity and inflammation. Cold Spring Harb Perspect Biol 2012. doi: 10.1101/cshperspect.a006049.

7. De Grove KC, Provoost S, Verhamme FM, et al. Characterization and Quantification of Innate 
Lymphoid Cell Subsets in Human Lung. PLOS ONE 2016;11:e0145961.

8. Mindt BC, Fritz JH, Duerr CU. Group 2 Innate Lymphoid Cells in Pulmonary Immunity and Tissue Homeostasis. Front Immunol 2018;9:840

9. Gordon S, Pluddemann A. Tissue macrophages: heterogeneity and functions. BMC Biol 2017;15:53.

10. Trapnell BC, Whitsett JA. Gm-CSF regulates pulmonary surfactant homeostasis and alveolar macrophage-mediated innate host defense. Annu Rev Physiol 2002;64:775-802.

11. Snelgrove RJ, Goulding J, Didierlaurent AM, et al. A critical function for CD200 in lung immune homeostasis and the severity of influenza infection. Nature Immunology 2008;9:1074.

12. Soltys J, Bonfield T, Chmiel J, et al. Functional IL-10 deficiency in the lung of cystic fibrosis (cftr(-/-)) and IL10 knockout mice causes increased expression and function of B7 costimulatory molecules on alveolar macrophages. J Immunol 2002;168:1903-10.

13. Meliopoulos VA, Van de Velde LA, Van de Velde NC, et al. An Epithelial Integrin Regulates the Amplitude of Protective Lung Interferon Responses against Multiple Respiratory Pathogens. PLoS Pathog 2016;12:e1005804.

14. Jiang Z, Zhou Q, Gu C, et al. Depletion of circulating monocytes suppresses IL-17 and HMGB1 expression in mice with LPS-induced acute lung injury. Am J Physiol Lung Cell Mol Physiol 2017;312:L231-42.

15. Rosseau S, Hammerl P, Maus U, et al. Phenotypic characterization of alveolar monocyte recruitment in acute respiratory distress syndrome. Am J Physiol Lung Cell Mol Physiol 2000;279:L25-35.

16. Johnston LK, Rims CR, Gill SE, et al. Pulmonary macrophage subpopulations in the induction and resolution of acute lung injury. American journal of respiratory cell and molecular biology 2012;47:417-26.

17. Wang F, Fu X, Wu X, et al. Bone marrow derived M2 macrophages protected against lipopolysaccharideinduced acute lung injury through inhibiting oxidative stress and inflammation by modulating neutrophils and $\mathrm{T}$ lymphocytes responses. Int Immunopharmacol 2018;61:162-8.

18. Tu GW, Shi Y, Zheng YJ, et al. Glucocorticoid attenuates acute lung injury through induction of type 2 macrophage. J Transl Med 2017;15:181.

19. Anderson BO, Brown JM, Shanley PF, et al. Marginating neutrophils are reversibly adherent to normal lung endothelium. Surgery 1991;109:51-61.

20. Bradley LM, Douglass MF, Chatterjee D, et al. Matrix metalloprotease 9 mediates neutrophil migration into the airways in response to influenza virus-induced toll-like receptor signaling. PLoS Pathog 2012;8:e1002641.

21. Keck T, Balcom JHt, Fernandez-del Castillo C, et al. Matrix metalloproteinase-9 promotes neutrophil migration and alveolar capillary leakage in pancreatitis-associated lung injury in the rat. Gastroenterology 2002;122:188-201.

22. Porto BN, Stein RT. Neutrophil Extracellular Traps in Pulmonary Diseases: Too Much of a Good Thing? Front Immunol 2016;7:311.

23. Narayana Moorthy A, Narasaraju T, Rai P, et al. In vivo and in vitro studies on the roles of neutrophil extracellular traps during secondary pneumococcal pneumonia after primary pulmonary influenza infection. Front Immunol $2013 ; 4: 56$.

24. Cortjens B, de Boer OJ, de Jong R, et al. Neutrophil extracellular traps cause airway obstruction during respiratory syncytial virus disease. J Pathol 2016;238:401-11.

25. Narasaraju T, Yang E, Samy RP, et al. Excessive neutrophils and neutrophil extracellular traps contribute to acute lung injury of influenza pneumonitis. Am J Pathol 2011;179:199-210.

26. Juneau RA, Pang B, Weimer KE, et al. Nontypeable Haemophilus influenzae initiates formation of neutrophil extracellular traps. Infect Immun 2011;79:431-8.

27. Liu S, Su X, Pan P, et al. Neutrophil extracellular traps are indirectly triggered by lipopolysaccharide and contribute to acute lung injury. Scientific Reports 2016;6:37252.

28. Caudrillier A, Kessenbrock K, Gilliss BM, et al. Platelets induce neutrophil extracellular traps in transfusion-related acute lung injury. J Clin Invest 2012;122:2661-71.

29. Semeraro F, Ammollo CT, Morrissey JH, et al. Extracellular histones promote thrombin generation through platelet-dependent mechanisms: involvement of platelet TLR2 and TLR4. Blood 2011;118:1952-61.

30. Hamid U, Krasnodembskaya A, Fitzgerald M, et al. Aspirin reduces lipopolysaccharide-induced pulmonary inflammation in human models of ARDS. Thorax 2017;72:971-80.

31. Kor DJ, Carter RE, Park PK, et al. Effect of Aspirin on Development of ARDS in At-Risk Patients Presenting to the Emergency Department: The LIPS-A Randomized Clinical Trial. JAMA 2016;315:2406-14.

32. Nie L, Wu W, Lu Z, et al. CXCR3 May Help Regulate the Inflammatory Response in Acute Lung Injury via a Pathway Modulated by IL-10 Secreted by CD 8 + CD122+ Regulatory T Cells. Inflammation 2016;39:526-33. 
33. Connors TJ, Ravindranath TM, Bickham KL, et al. Airway CD8(+) T Cells Are Associated with Lung Injury during Infant Viral Respiratory Tract Infection. Am J Respir Cell Mol Biol 2016;54:822-30.

34. Sakaguchi R, Chikuma S, Shichita T, et al. Innate-like function of memory Th17 cells for enhancing endotoxininduced acute lung inflammation through IL-22. Int Immunol 2016;28:233-43.

35. Muir R, Osbourn M, Dubois AV, et al. Innate Lymphoid Cells Are the Predominant Source of IL-17A during the Early Pathogenesis of Acute Respiratory Distress Syndrome. Am J Respir Crit Care Med 2016;193:407-16.

36. Li JT, Melton AC, Su G, et al. Unexpected Role for Adaptive alphabetaTh17 Cells in Acute Respiratory Distress Syndrome. J Immunol 2015;195:87-95.

37. Wang L, Wang X, Tong L, et al. Recovery from acute lung injury can be regulated via modulation of regulatory $\mathrm{T}$ cells and Th17 cells. Scand J Immunol 2018;88:e12715.

38. Xiong Y, Wang J, Wei D, et al. Prognostic values of Th17 cells level in bronchoalveolar lavage fluid in children of sepsis with acute lung injury. Zhongguo Dang Dai Er Ke Za Zhi 2015;17:942-5.

39. Holloway TL, Rani M, Cap AP, et al. The association between the Th-17 immune response and pulmonary complications in a trauma ICU population. Cytokine 2015;76:328-33.

40. Pociask DA, Scheller EV, Mandalapu S, et al. IL22 is essential for lung epithelial repair following influenza infection. The American journal of pathology 2013;182:1286-96.

41. Kapur R, Kim M, Aslam R, et al. T regulatory cells and dendritic cells protect against transfusion-related acute lung injury via IL-10. Blood 2017;129:2557-69.

42. Li GG, Cao YH, Run Y, et al. Inhibition of CD8(+) T cells and elimination of myeloid cells by CD4(+) Foxp3(-) T regulatory type 1 cells in acute respiratory distress syndrome. Clin Exp Pharmacol Physiol 2016;43:1191-8.

43. Yu ZX, Ji MS, Yan J, et al. The ratio of Th17/Treg cells as a risk indicator in early acute respiratory distress syndrome. Crit Care 2015;19:82.

44. Ronit A, Plovsing RR, Gaardbo JC, et al. T cell subsets in human airways prior to and following endobronchial administration of endotoxin. Respirology 2015;20:579-86.

45. Mock JR, Garibaldi BT, Aggarwal NR, et al. Foxp3+ regulatory $\mathrm{T}$ cells promote lung epithelial proliferation. Mucosal Immunol 2014;7:1440-51.

46. D'Alessio FR, Tsushima K, Aggarwal NR, et al.
CD4+CD25+Foxp3+ Tregs resolve experimental lung injury in mice and are present in humans with acute lung injury. J Clin Invest 2009;119:2898-913.

47. Aggarwal NR, D'Alessio FR, Tsushima K, et al. Regulatory $T$ cell-mediated resolution of lung injury: identification of potential target genes via expression profiling. Physiol Genomics 2010;41:109-19.

48. Dial CF, Tune MK, Doerschuk CM, et al. Foxp3(+) Regulatory T Cell Expression of Keratinocyte Growth Factor Enhances Lung Epithelial Proliferation. Am J Respir Cell Mol Biol 2017;57:162-73.

49. Singer BD, Mock JR, Aggarwal NR, et al. Regulatory T cell DNA methyltransferase inhibition accelerates resolution of lung inflammation. Am J Respir Cell Mol Biol 2015;52:641-52.

50. Bai J, Qiu SL, Zhong XN, et al. Erythromycin enhances $\mathrm{CD} 4+$ Foxp3 + regulatory $\mathrm{T}$-cell responses in a rat model of smoke-induced lung inflammation. Mediators Inflamm 2012;2012:410232.

51. Li Q, Hu X, Sun R, et al. Resolution acute respiratory distress syndrome through reversing the imbalance of Treg/Th17 by targeting the cAMP signaling pathway. Mol Med Rep 2016;14:343-8.

52. McGrath-Morrow SA, Lee S, Gibbs K, et al. Immune response to intrapharyngeal LPS in neonatal and juvenile mice. Am J Respir Cell Mol Biol 2015;52:323-31.

53. Tang L, Bai J, Chung CS, et al. Active players in resolution of shock/sepsis induced indirect lung injury: immunomodulatory effects of Tregs and PD-1. J Leukoc Biol 2014;96:809-20.

54. Tang L, Bai J, Chung CS, et al. Programmed cell death receptor ligand 1 modulates the regulatory $T$ cells' capacity to repress shock/sepsis-induced indirect acute lung injury by recruiting phosphatase SRC homology region 2 domain-containing phosphatase 1. Shock 2015;43:47-54.

55. Zhou Y, Tang L, Lin M, et al. Expression of cytotoxic T-lymphocyte antigen 4 on CD4+ and CD8+ T cells is increased in acute lung injury. DNA Cell Biol 2013;32:722-6.

56. Laffey JG, Matthay MA. Fifty Years of Research in ARDS. Cell-based Therapy for Acute Respiratory Distress Syndrome. Biology and Potential Therapeutic Value. Am J Respir Crit Care Med 2017;196:266-73.

57. Hayes M, Masterson C, Devaney J, et al. Therapeutic efficacy of human mesenchymal stromal cells in the repair of established ventilator-induced lung injury in the rat. Anesthesiology 2015;122:363-73.

58. Rojas M, Cardenes N, Kocyildirim E, et al. Human 
adult bone marrow-derived stem cells decrease severity of lipopolysaccharide-induced acute respiratory distress syndrome in sheep. Stem Cell Res Ther 2014;5:42.

59. Lee JW, Krasnodembskaya A, McKenna DH, et al. Therapeutic effects of human mesenchymal stem cells in ex vivo human lungs injured with live bacteria. Am J Respir Crit Care Med 2013;187:751-60.

60. Krasnodembskaya A, Song Y, Fang X, et al. Antibacterial effect of human mesenchymal stem cells is mediated in part from secretion of the antimicrobial peptide LL-37. Stem Cells 2010;28:2229-38.

61. Wilson JG, Liu KD, Zhuo H, et al. Mesenchymal stem (stromal) cells for treatment of ARDS: a phase 1 clinical trial. Lancet Respir Med 2015;3:24-32.

62. Matthay MA, Calfee CS, Zhuo H, et al. Treatment with allogeneic mesenchymal stromal cells for moderate to severe acute respiratory distress syndrome (START study): a randomised phase 2a safety trial. Lancet Respir Med 2019;7:154-62.

63. Wanke-Jellinek L, Keegan JW, Dolan JW, et al. Characterization of lung infection-induced TCR $\gamma \delta$ T cell phenotypes by CyTOF mass cytometry. Journal of Leukocyte Biology 2016;99:483-93.

64. Spitzer MH, Nolan GP. Mass Cytometry: Single Cells, Many Features. Cell 2016;165:780-91.

65. Morrell ED, Wiedeman A, Long SA, et al. Cytometry TOF identifies alveolar macrophage subtypes in acute respiratory distress syndrome. JCI Insight 2018;3.

66. Kan M, Shumyatcher M, Himes BE. Using omics approaches to understand pulmonary diseases. Respir Res 2017;18:149.

67. Xu Y, Mizuno T, Sridharan A, et al. Single-cell RNA sequencing identifies diverse roles of epithelial cells in idiopathic pulmonary fibrosis. JCI Insight 2016;1:e90558.

Cite this article as: Wong JJM, Leong JY, Lee JH, Albani S, Yeo JG. Insights into the immuno-pathogenesis of acute respiratory distress syndrome. Ann Transl Med 2019;7(19):504. doi: $10.21037 /$ atm.2019.09.28 